\title{
Privacy-Preserving Ride Sharing Scheme with Global Social Network for Vehicles Using Big Data
}

\author{
R. Vignesh*, B. Keerthi Samhitha, C. M. Suja and S. Divya \\ Department of C.S.E, Sathyabama Institute of Science and Technology, Chennai - 600119, Tamil Nadu, India; vi- \\ gnesh.cse@sathyabama.ac.in, samhitha711@gmail.com,cmsuja@gmail.com,divyachennai2017@gmail.com
}

\begin{abstract}
Objectives: To built up a productive ride sharing come auto pooling application which empowers the client to go to their goals on the fly easily and reasonable subject to time, limit, and money related limitations, guaranteeing the protection and security concerns and guarantees the client for playing it safe on the goal relying upon the customized setting mindful wellbeing levels. Methods: Taxi-sharing framework acknowledges taxi travelers' ongoing ride demands sent from advanced mobile phones. It plans appropriate taxicabs to get them through ridesharing and private riding, subject to time limit and fiscal requirements. An auto pooling choice for private auto proprietors whoever goes in a customary course. A customized setting mindful security forecast and proposal for goal area is accommodated by guaranteeing precautionary measures. Findings: This plan can accomplish alluring security highlights. Consequently, we built up a productive ridesharing come auto pooling application which empowers the client to go to their goals on the fly easily and reasonable subject to time, limit, and money related limitations, guaranteeing the protection and security concerns and guarantees the client for playing it safe on the goal relying upon the customized setting mindful wellbeing levels. Improvements: Distinctive instances of ride sharing can be improved out by our plan with considering the clients' inclinations.
\end{abstract}

Keywords: CI Values, Global Social Networks (GSN), Ride Sharing, Taxi Sharing Framework

\section{Introduction}

In the course of recent years, the vehicle business has made imperative jumps in conveying computerization to auto driving ${ }^{1}$. Self-sufficient Vehicles (AVs) are outfitted with cutting edge detecting and relational abilities, route gadgets, PC vision innovation, and so on. To empower the vehicles to self-sufficiently drive themselves with no mediation from people. AVs can possibly generally propel transportation frameworks by diminishing blasts, helping movement streams, and lessening travel time. Be that as it may, the innovation utilized as a part of AVs is costly and the AVs' cost will be high. Since AVs can drive themselves, they may not be private gadgets any longer, but rather on-request benefit. As of now individuals utilize possess autos yet later on as opposed to owning an $\mathrm{AV}$, many individuals can arrange an AV from a taxicab organization when they require. This is an intriguing and promising approach to ease the high cost issue of the AVs. Such on-request administration will be viable and mainstream in AVs because of the rejection of the human driver exertion. Ride-sharing (or carpooling) permits AVs to be shared by clients. For e.g., to share the cost of on-request taxicab benefit. Since AV scan drive them, they will make ride sharing suitable, prominent, and in some cases important. In any case, to compose ride sharing, clients need to uncover not just their treks' get/dropoff areas, knowing the time a man will leave home is for the most part valuable data to the group of criminals.

In this study, we propose a protection saving plan to build up ride sharing. To our colleague, existing security saving strategies ${ }^{2}$ can't be connected effectively and proficiently in ride sharing because of the interesting issues and prerequisites. Additionally, concealing the clients'

*Author for correspondence 
distinctions ${ }^{2}$ is insufficient in light of the fact that aggressors can analyze the clients from their get/drop-off areas. We utilize a gathering mark plan, for example, our proposition in ${ }^{3}$, to guarantee clients secrecy. We likewise utilize a likeness estimation procedure over scrambled information, for example ${ }^{4}$, to empower a server to gauge the similitude of the clients' excursion measurements without knowing the information. Once the server finds a client who can share ride, it sends the client's mark to the AV client who can follow the mark to the underwriter's character. Our plan considers different cases for ride sharing and permits clients to prescribe their inclinations, for example, the most extreme separation between excursions begin/end areas and a client's get/drop-off areas.

Examination has affirmed that the proposed plan can arrange ride sharing without uncovering touchy data. We have executed the plan utilizing Visual $\mathrm{C}$ on a genuine guide and measured execution measurements. The outcomes have set up that the correspondence and capacity overhead is worthy and the inquiry time to sort out a mutual ride is little.

\section{Survey}

\subsection{Spatial Transformation}

In $^{5}$ anticipated Hierarchical Space Division (HSD), ErrorBased Transformation (ERB) and $\mathrm{HSD}^{*}$ by inspecting the benefit of HSD and ERB. The HSD ensures information by utilizing a spatial separating procedure for redistributing the changed information. This technique is solid against the assault done by enemies who have the subset of database data yet weak against the individuals who have an objective appropriation. The ERB brings limited blunders into the information that is reversible with the assistance of the safe hash work. The ERB change is feeble against the individuals who have foundation learning of information dissemination. They additionally proposed $\mathrm{HSD}^{*}$ by joining HSD and ERB. Be that as it may, since the spatial remodel system keeps up directions of POIs, it is risky when the challenger has foundation information of unique POI conveyance and some piece of database.

\subsection{Distance-Oriented Transformation}

\section{Based Methods}

$\operatorname{In}^{5}$ anticipated the separation arranged change methods called MPT (Metric Preservation Technique). This technique changes over a remarkable spatial record in a metric space into another metric space datasets by utilizing separation between POIs. In this way, the information proprietor changes his information objects into a separation based metric dataset and subcontracts the changed over database to a specialist organization. In this manner, the specialist co-op can't attempt the first arrange of POIs while the question handling calculation guarantees the right answer. The server figures a list structure on the scrambled dataset to encourage proficient inquiry. For question preparing, the information proprietor educates each client of the change. At inquiry time, a dependable client applies the change work (with a key) to the question and sends the changed question to the server. At that point, the server forms the question and reports the outcomes back to the client. Eventually, the client deciphers the recovered outcomes again into the genuine outcomes. Figure 1 (left) demonstrates the case of grapple hubs and its POIs. Acknowledge that there are 2 grapple hubs and 8 POIs around them. The Table in Figure 1 clarifies the changed POI list by utilizing the OPES for every POI in stays. The p1, p3, p4, p5 are appointed to grapple 1, and others allotted to stay 2 . The changed database stores the request rationing encoded remove amongst stay and POI.

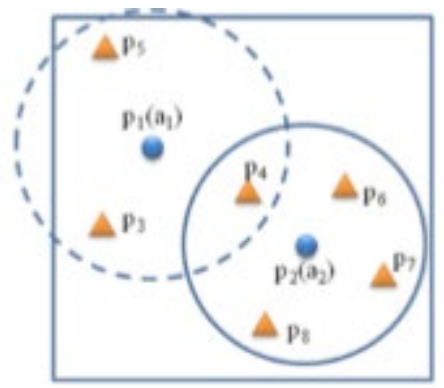

\begin{tabular}{|c|c|c|c|c|c|}
\hline ID & $\begin{array}{c}\text { Anchor } \\
\text { ID }\end{array}$ & Distance & ID & $\begin{array}{c}\text { Anchor } \\
\text { ID }\end{array}$ & Distance \\
\hline 1 & 1 & $\mathrm{OPE}(0.00)=0.00$ & 5 & 1 & $\mathrm{OPE}(0.32)=0.80$ \\
2 & 2 & $\mathrm{OPE}(0.00)=0.00$ & 6 & 2 & $\mathrm{OPE}(0.10)=0.20$ \\
3 & 1 & $\mathrm{OPE}(0.35)=1.00$ & 7 & 2 & $\mathrm{OPE}(0.18)=0.40$ \\
4 & 1 & $\mathrm{OPE}(0.20)=0.60$ & 8 & 2 & $\mathrm{OPE}(0.34)=0.90$ \\
\hline
\end{tabular}

Figure 1. Anchors and POI distribution and encrypted POI index. 


\subsection{Privacy-Preserving Semantic Multi-Keyword Ranked Search}

The issue of multi-watchword dormant semantic positioned seek over scrambled cloud information and recovering the most important documents is comprehended here. We characterize another plan named Latent Semantic Analysis (LSA) based multi-catchphrase positioned seek which arrangements multi-watchword idle semantic positioned look. By utilizing LSA, the anticipated plan could return the correct coordinating documents, as well as the records including the terms dormant semantically identified with the question watchword. For instance, when the client inputs the watchword "Car" to pursuit documents, the expected technique returns the records containing "Car", as well as the records with the expression "Auto". We take a vast framework of termreport proposal information and develop a semantic space wherein terms and archives are firmly connected are set close to each other. To meet the test of supporting such multi-watchword semantic without protection breaks, we propose the thought: The Multi-Keyword Ranked Search (MRSE) utilizing "Dormant Semantic Analysis". In ${ }^{6}$ we investigate the potential cyber attacks specific to automated vehicles, with their special needs and vulnerabilities. We analyze the threats on autonomous automated vehicles and cooperative automated vehicles

\subsection{Multi-Keyword Ranked Search with Anonymous ID Assignment}

Since a cloud information facilitating administration including three unique substances, the information proprietor, the information client alongside his ID, and the cloud server. The information proprietor first records on cloud utilizing namelessness calculation for distributed computing administrations. Before redeemable client enrollment data to database show on cloud mysterious calculation prepare the information and after that unknown information is spared to record-keeping database. The information proprietor has an accumulation of information records to be outsourced to the cloud server in the encoded frame. To empower looking capacity over compelling information application, the information proprietor, will first form a scrambled searchable file before outsourcing, and afterward subcontract both the list and the encoded archive accumulation to the cloud server. The work manages efficacious calculations for doling out identifiers (IDs) to the clients on the cloud in a manner that the IDs are unknown utilizing a scattered calculation with no focal specialist. Given are $\mathrm{N}$ hubs, this task is generally a change of the numbers $\{1 \ldots \mathrm{N}\}$ with every ID being known just to the hub to which it is designated. Our principle calculation depends on a technique for secretly sharing basic information and results in strategies for strong sharing of complex information. To scan the report gathering for given catchphrases, an endorsed client having an ID procures a relating trapdoor $\mathrm{T}$ through pursuit control components, for instance, communicate encryption. On accepting $\mathrm{T}$ from an information client, cloud server is liable to look the list and afterward gives back the relating set of scrambled archives. To advance the report recovery precision, the query output ought to be positioned by the cloud server offering to some positioning criteria (e.g., organize coordinating) and doling out mysterious $\mathrm{ID}^{3}$ to the client on cloud to make the information on cloud more secured. Also, to lessen the cost of correspondence the information client may send an elective number alongside the trapdoor $\mathrm{T}$ so that the cloud server just sends back top-k archives that are most relevant to the inquiry question. Finally, the get to control component is attempting to oversee unscrambling skills given to clients and the information gathering can be refreshed as far as embeddings new archives, advising existing ones, and erasing the current reports. $\operatorname{In}^{7}$ statistics on no of iterations has been calculated using Markov chain. In ${ }^{8}$ we first define a hotspot phenomenon that causes an obvious inconsistency in the network traffic pattern due to the large volume of packets originating from a small area. Second, we develop a realistic adversary model, assuming that the adversary can monitor the network traffic in multiple areas, rather than the entire network or only one area

\section{Proposed System}

We propose an ongoing taxi sharing application which will ping the travelers quite far in a period and cost effective route both to travelers and taxis. Protection will be held to the travelers relying upon their inclination by giving individual ride as well. With respect to as security is concerned each taxi clients' portable application has an alternative to report any sort of extra, which will send SMS and Mail to alarms to enrolled versatile numbers in a brisk and simple way. The server will likewise dispatch 
a message to administrator individuals with the GPS area of the taxi where the crisis was incited. Additionally, we present and acclimatize an auto surveying application for private auto proprietors who need to share their vehicle amid their general rides. Generally, most auto proprietors track a customary way on a dull premise. Numerous different clients who go to a similar area in the meantime can be gathered on a typical assertion between auto proprietor and the explorer. Add up to cost spent on the ride can be basic in view of the travel separation and time spent for the ride. Each taxi ride with a portable ought to be enlisted to our application self-governing relying upon the part of the client. Protection of every client will be kept up by giving pseudo character and individual data are kept secure and won't be imparted to different clients for any reasons substitute versatile numbers are utilized to trigger any sort of crisis and the ride status will be dispatched on social applications as you ask for to be posted on your rides.

For wellbeing, customized security gauges for client the wellbeing and wrongdoing file are computed in light of the direction. The objective of characterizing wrongdoing and wellbeing records is to furnish clients with security suggested data. Individuals are however not similarly presented and defenseless against all wrongdoing sorts. Age, sex and a variety of individual elements, inclinations and decisions assume a focal part in the understanding of an individual's security. Since such data may not be promptly available, we use rather the restriction abilities of a client's cell phone to sometimes record and locally store her direction follow. This empowers us to characterize the wrongdoing list level with which a client is agreeable, i.e., the normal wrongdoing list of the spots in her direction. At the point when enough wrongdoing data exists to empower the expectation of the not so distant future wrongdoing file of an area, we present the idea of customized security: the client is protected if her agreeable wrongdoing file level equivalents or surpasses the anticipated wrongdoing file of her present area. Operations are clarified in Figure 2.

\section{Modules}

\subsection{Various Roles of Users and Web Crawling}

There are four parts in our application, (1) Administrator, (2) Traveler, (3) Auto pooler and (4) Cab driver. Every client ought to be enrolled with our application aside from

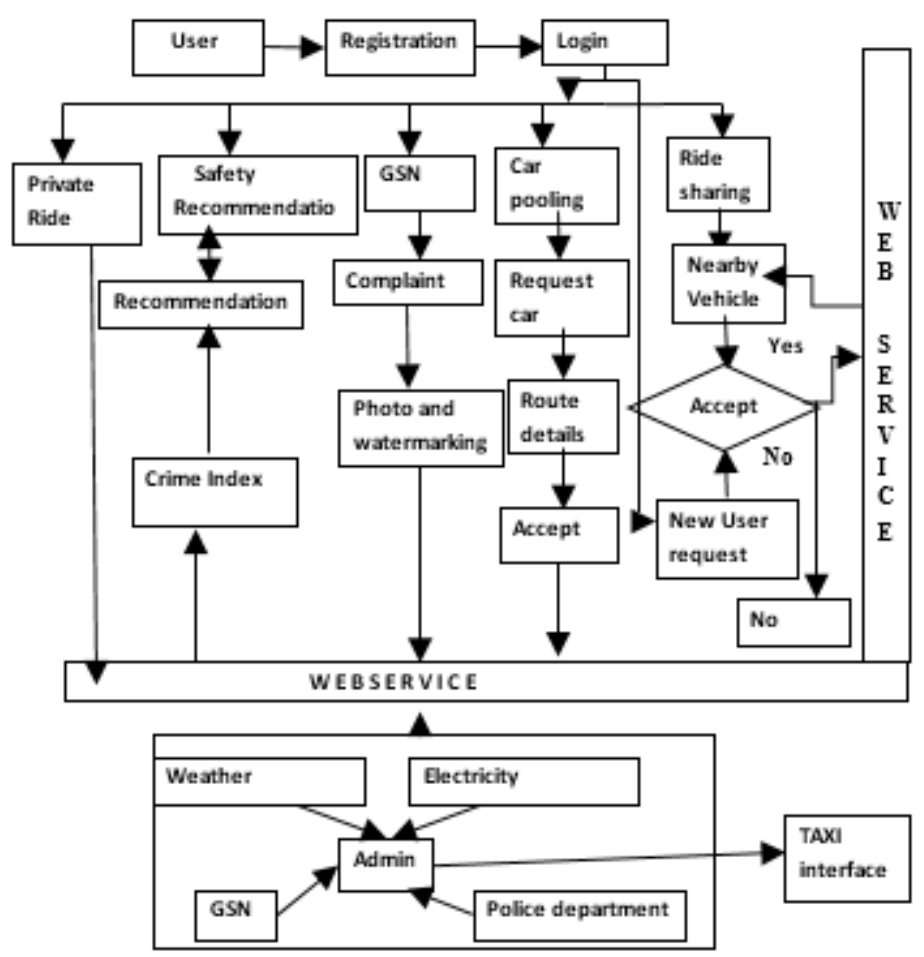

Figure 2. Architecture diagram. 
Admin as he approves client enlistment and produces pseudo personality. To begin with cab driver and carpooler are enlisted with their vehicle recognizable proof numbers and versatile numbers. After check is done the vehicle can be followed back by our application whenever. Taxi proprietors can refresh the bustling state and free condition of their taxi's as when a ride is begun or wrapped up. Web slithering is a procedure of separating information from different web applications through get to control and flexibilities for area based expectation to be finished by specialist co-op. All data for all spots ought to be known. The data contain control cut timetables from power office, condition factors from climate division, and criminal records from police office. Every division is unmistakable web applications and they have their own particular administrator level individuals safeguarding the datasets. Specialist co-op ought to ask for the different divisions so that the information's can be left from different web servers through web administrations. So a demand will be sent freely to every division and it will render as notices on the administrator pages. On the off chance that they acknowledge the solicitations sent from our specialist co-op we can access the web applications datasets.

\subsection{Building Real Time Taxi Sharing Application and GSN}

Administrator ought to set up application by putting vehicles in maps. No of vehicles are set on maps to show demo. As client login to the application he should pick whether he needs to travel private (alone) or needs a mutual ride. At that point the client must pick the source, goal and ought to ask for the administration. Client current position will be set apart on the guide as and when client enters the application.

We construct and incorporate a Global Social Network as in Figure 2 which empowers to foresee issues that the client may confront on an area, by assessing other client protests around there. All clients can post grumblings through GSN by taking photos of the issue they confronted over yonder. Our applications consequently implant the GPS values, date and time the snap was taken in the picture. A little clarification can likewise be given so that the administrator can without much of a stretch recognize the dissension and the watermark guarantee the accuracy of the issue. The $\mathrm{Db}$ will be effective in light of that and any client can manhandle a grievance. On the off chance that numerous clients mishandle a dissension it is distinguished as fake and killed in this manner making our application one of a kind. Along these lines, the administratorhas the specialist to hinder a client who abuses the protests.

The visitor client is given the office of review the grumblings posted by alternate clients in that area. The visitor client does not have to enlist with the application is to see the objections

\subsection{Taxi Searching and Ride Sharing}

The server will try to locate the nearby taxi by an efficient search process and finds a best match to the user requirement and will dispatch for pick up. A brief charge sheet is given for the ride request and the ride cost will be manipulated accordingly as the ride is finished. If the request type is a ride sharing request then the passengers can be grouped by mutual acknowledgements and can be accommodated based on time, space and monetary obliges. The passengers inside the car will be requested to give permission for a new comer who is requesting a ride share service. If and only if all the passengers within the car accept the request the taxi driver is intimated to pick up the new passenger.

\subsubsection{Searching Algorithm}

Consider the below instance diagram shown in the Figure 3 for description of the searching algorithm that is mainly used here is explained.

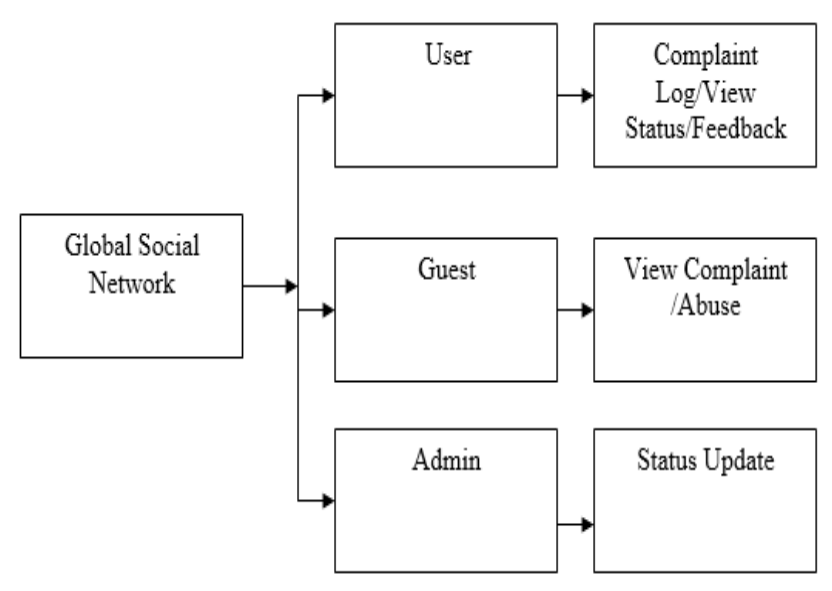

Figure 3. Operations of GSN. 
Consider the underneath example graph appeared in the Figure 4 for portrayal of the seeking calculation that is primarily utilized here is clarified. Accept that there is a demand $r$ and the present time Tour the lattice cell $g 7$ is where Qo (get point). The main matrix cell to be found and chose is g7 here. Some other cell would be chosen irregular by the seeking calculation only on the off chance that it holds Equation (1). To quickly discover every one of the frameworks that the Equation (1) holds, the taxi examining calculation first tests all cells in the lattices per the request in the rundown protected by spatial worldly record and finds the primary network cell gfi which neglects to hold Equation (1).

$$
\text { Ti7+tcurr } \leq \text { Qo }
$$

Where Ti7 speaks to the travel time between lattice cell g7 and gi. After a taxi is begun utilizing the inquiry calculation, a brief charge leaf is given for the ride ask for and the ride cost will be controlled as needs be the point at which the ride is done. In the event that the demand shape is a ride sharing solicitation then the travelers can be assembled by joint affirmations and can be suited in light of time, space and money related obliges. The travelers inside the auto will be asked for to give proceed for another comer who is wishing a ride share benefit. On the off chance that and just if every one of the travelers inside acknowledges the demand the cab driver is insinuated to get the new traveler.

\subsection{Car Pooling and Bill Generation}

A different procedure is created to bolster auto pooling for normal course clients and the proprietor's courses are refreshed on their enrollment procedure as in Figure 4. An auto pooling solicitation can be given by a client by saying the course subtle elements that ought to be taken after routinely. The server will locate a legitimate auto pooler and will advise both carpoolers and requesters. On the off chance that both acknowledge the proposition, then they will be pinged and followed by your application. Bill will be produced in light of the sort of travel, travel separation and time in view of the duty said while reserving for a ride. Security can be accomplished by having crisis triggers and by posting the travel data on social Medias. Security will be held to the travelers relying upon their inclination by giving individual ride and utilizing pseudo characters.

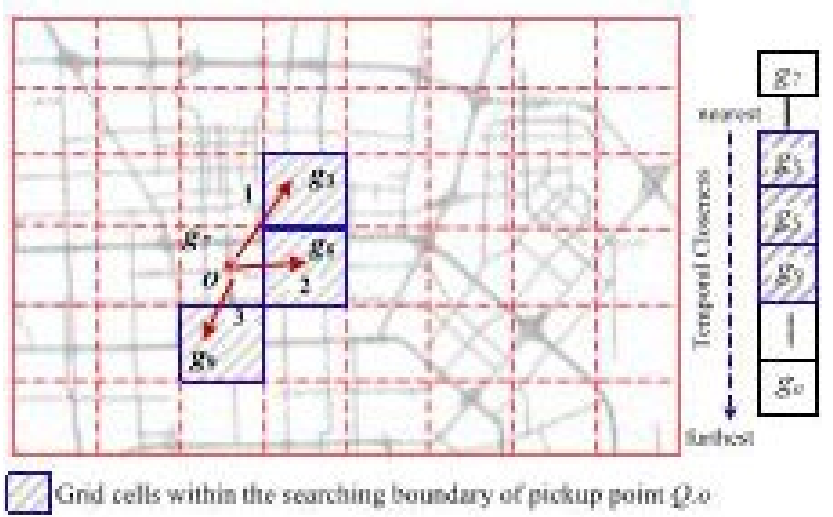

Figure 4. Taxi searching algorithm.

\subsection{Personalized Context Aware Safety Prediction and Recommendation}

Security data however is most valuable when accommodated the present or not so distant future. The client directions have a vital influence in anticipating the qualities which are separated from the direction hint of client. The specialist organization predicts the security levels if enough forecast qualities are accessible for future area relying upon GSN, police records, climate records, and power cut calendars. Client direction proposal recorded by his versatile application and the GSN follow helps foreseeing the wrongdoing file level with which the client is agreeable. The normal wrongdoing file estimations of client's direction must be equivalent or more noteworthy than the alongside future area wrongdoing list values. The CI values relies on upon the weight age of violations pro-

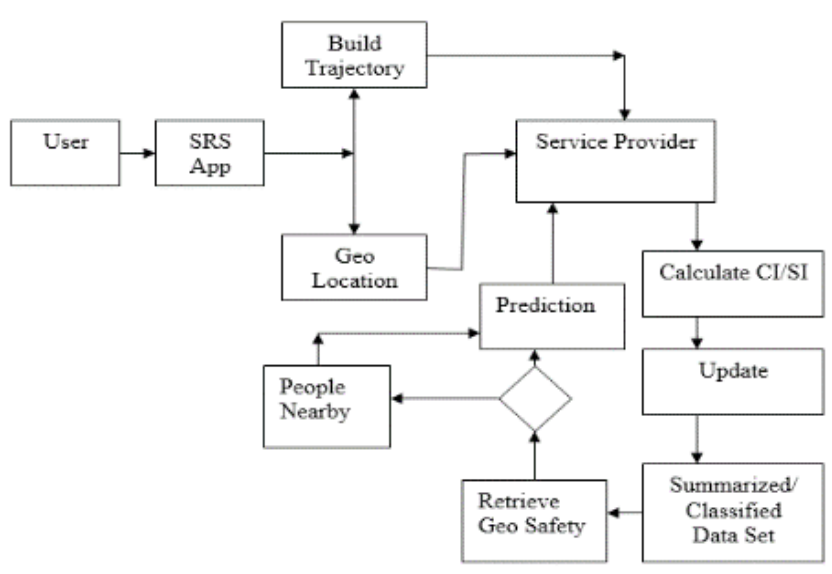

Figure 5. Context aware safety prediction and recommendation. 
vided details regarding that areas. The expectation is done in 3 levels demonstrating red, yellow, green in view of the security forecast through direction follow which manages the client in his goal area and also ensures he or she is playing it safe in that area as shown in Figure 5.

\section{Conclusion}

We have proposed a security saving ride sharing plan for AVs. Distinctive instances of ride sharing can be sorted out by our plan with considering the clients' inclinations. Our investigation has shown that our plan can accomplish alluring security highlights. Consequently, we built up a productive ridesharing cum auto pooling application which empowers the client commute easily with reasonable time and money limitations and security concerns.

\section{References}

1. Ahmed BT, Sheriff, Rabies K, Mahmud M, Liang X. Privacy-preserving ride sharing scheme for autonomous vehicles in big data era. IEEE Internet of Things Journal. 2017; 4(2):611-8. crossref
2. Shao J, Lin X, Lu R, Zuo C. A threshold anonymous authentication protocol for vanets. IEEE Transactions on Vehicular Technology. 2016; 65(3):1711-20. crossref

3. Liang X, Cao Z, Shoo J, Lin H. Short group signature without random oracles. Information and Communications Security. 2007; 4861:69-82.

4. Cao N, Wang C, Li M, Ran K, Lou W. Privacy-preserving multi-keyword ranked search over encrypted cloud data. IEEE Transactions on Parallel and Distributed Systems. 2014; 25(1):222-33. crossref

5. Yij ML, Assent I, Jensen CS, Kilns P. Outsourced Similarity Search on Metric Data Assets. IEEE Transactions on Knowledge and Data Engineering. 2012; 24(2):338-52. crossref

6. Petit J, Shaver SE. Potential cyber attacks on automated vehicles. IEEE Transactions on Intelligent Transportation Systems. 2015; 16(2):546-56.

7. Dunning LA, Kerman R. Privacy preserving data sharing with anonymous ID assignment. IEEE Transactions on Information Forensics and Security. 2013; 8(2):402-13.

8. Mahmud M, Shin X. Cloud-based Scheme for protecting source location privacy against hotspot-locating attack in wireless sensor networks. IEEE Transactions on Parallel and Distributed Systems. 2012; 23(10):1805-18. crossref 